\title{
Cardiac reserve mobilization trend during exercise and recovery after exercise
}

\author{
Leichu Liu ${ }^{1}$, Xiaobo Yan ${ }^{1 *}$, Shouzhong Xiao ${ }^{2}$, Suyuan Deng ${ }^{1}$, Cong Zhang ${ }^{1}$, Linmei Luo ${ }^{1}$ \\ ${ }^{1}$ Institute of Physical Education, Chongqing University, Chongqing, China \\ ${ }^{2}$ Bo-Jing Medical Informatics Institute, Chongqing, China \\ Email: *xbyan@cqu.edu.cn
}

Received 1 May 2013; revised 29 May 2013; accepted 12 June 2013

Copyright (c) 2013 Leichu Liu et al. This is an open access article distributed under the Creative Commons Attribution License, which permits unrestricted use, distribution, and reproduction in any medium, provided the original work is properly cited.

\begin{abstract}
Objective: Little is known about the cardiac contractility recovery after exercise. The objective of the study was to explore a method to evaluate the extent and speed of cardiac function up-regulation during exercise and the recovery course of cardiac contractility and heart rate after exercise. Methods: Ten student athletes and ten student non-athlete voluntarily participated in this controlled study. Three indicators were selected: 1) amplitude ratio of the first to second heart sound (S1/S2); 2) heart rate (HR); 3) power output (W). Phonocardiogram exercise test (PCGET) was adopted. A four-stage workload increment protocol was used. Phonocardiograms were recorded in the sitting position at rest and immediately after each test stage. The time taken for completing the workloads $1750 \mathrm{~J}, 3500 \mathrm{~J}, 5250 \mathrm{~J}$, and $7000 \mathrm{~J}$ was recorded, respectively. During recovery heart sound signals were recorded immediately after exercise, and at 1,5 , $10,15,20,25$, and 30 minutes after exercise. S1/S2, $\mathrm{HR}$, and $\mathrm{W}$ were calculated from the measured data. Cardiac function change trend graphs were constructed. Results: During exercise, HR and S1/S2 ratio increased with the increase in workload from 1750 $\mathrm{J}$ to $7000 \mathrm{~J}$; the level and speed of increase in power output and S1/S2 ratio of the athletes were higher than the general students; power done by the general students decreased earlier than the athletes. During recovery course, the recovery course of the general students was slower than the athletes. Conclusion: This method for evaluating cardiac function up-regulation and recovery course is safe, easy, reliable, and effective, which is beneficial for selecting athletes, training, and matchmaking.
\end{abstract}

Keywords: Cardiac Function; Amplitude Ratio of the "Corresponding author.
First to Second Heart Sound (S1/S2); Power Output; Up-Regulation; Graph

\section{INTRODUCTION}

Exercise capacity is closely associated with survival and mortality [1,2] The study by Myers et al. [2] showed that each 1-MET increase in exercise capacity conferred a 12 percent improvement in survival. Consistently, another study [1] demonstrated that exercise capacity is an independent predictor of all-cause mortality in older men; the relationship is inverse and graded, with most survival benefits achieved in those with an exercise capacity $>5$ METs; survival improved significantly when unfit individuals became fit. Cardiac reserve is one of the most important physiological bases of exercise capacity. There is a 20 -fold difference between the most impaired cardiac function and that of the fittest person [3].

Cardiac contractility reserve (CCR) and heart rate reserve (HRR) are reflected in the dynamic change of the heart status, therefore, the evaluation of CCR and HRR is conducted in various stress tests. However, cardiac function is not only reflected in its up-regulation, but also in the recovery course. Previous studies have mainly focused on the cardiac function up-regulation, having neglected the recovery course. Even if some studies are involved in the recovery course, they are mainly in heart rate recovery [4,5], little in cardiac contractility recovery.

This study tried to explore a method to evaluate the extent and speed of cardiac function up-regulation during exercise and the recovery course of cardiac contractility and heart rate after exercise.

The responses of the heart to exercise include many aspects. Three indicators to evaluate the changes of cardiac function during exercise testing have been selected.

1) Amplitude ratio of the first to second heart sound (S1/S2): One feature of the heart sounds that is a direct 
manifestation of the left ventricular contractile state and may be used for rapid noninvasive detection of left ventricular systolic function is the first heart sound (S1). Previous studies have shown that there is a very close relationship between the amplitude of the first heart sound and cardiac contractility [6,7]. A study by Hansen et al. [7] showed that changes in the amplitude of the first heart sound are closely related to the maximum rate of rise of left ventricular pressure $(r=0.9551, P<0.001)$. Therefore, the change trend of the amplitude of the first heart sound (S1) can be used to evaluate cardiac reserve and cardiac endurance. Since the amplitude of S2 is a reflection of the peripheral resistance [8], S1/S2 ratio reflects the relationship between the cardiac contractility and the peripheral resistance, including the regulatory capacity of the myocardium under stress. The study by Hsieh et al. demonstrated that the relative amplitudes of S1 and S2 (aortic component of second heart sound) represent left ventricular $\mathrm{dP} / \mathrm{dt}$ and ejection fraction (EF) in humans, and S1, corrected for S2, is decreased in patients with impaired LV systolic function [9];

2) Heart rate (HR): Simultaneous measurement of both cardiac contractility change trend and heart rate change trend after exercise might be beneficial to comprehensive evaluation of cardiac function [10];

3) Power output during exercise: Power output is an important indicator for evaluating exercise performance of both athletes [11,12] and patients [13,14]. Cardiac power output at peak exercise was found to be significantly related to aerobic capacity. One of the best measures of athletic performance is power output during exercise. There is a 20 -fold difference between the most impaired cardiac function and that of the fittest subject [3].

In this paper the method for evaluating cardiac reserve mobilization trend during exercise and recovery after exercise is described and the preliminary results of the study are analyzed.

\section{SUBJECTS AND METHODS}

\subsection{Subjects}

Ten non-athletes (seven males and three females, aged from 19 to 21 years, Group A) and ten athletes (nine males and one female, aged from 14 to 17 years, Group B) were voluntarily enrolled in this study. The inclusion criteria were: 1 ) age was less than 25 years old; 2) giving informed consent; 3) free of any cardiovascular diseases and free of any other serious diseases. The exclusion criteria were: 1) on any medication; 2) presence of any contraindications to physical exercise. All subjects signed an informed consent form approved by the Ethics Committee of Institute of Physical Education, Chongqing University.

\subsection{Instruments}

Cardiac Contractility Monitor (CCM, developed by BoJing Medical Informatics Institute, Chongqing, China) was used for this investigation. The hardware of CCM consists of a phonocardiographic sensor, a heart sound signal preprocessing box, a computer, and a printer. CCM uses a sampling rate of $8 \mathrm{kHz}$ with 8 bits per sample (monochannel). The software includes a fundamental heart sound measurement system, the developing environment of which is Visual Basic 6.0 (Microsoft Inc,). The application's target operating platform is Windows XP.

\subsection{Exercise Testing}

Since exercise can simultaneously cause a significant change in the inotropic and chronotropic status of the heart, phonocardiogram exercise test (PCGET) was adopted $[15,16]$ in this study. Examination was started after the subject rested for 15 minutes. A PCG sensor was placed on the subjects' pericardium. Phonocardiograms were recorded in the sitting position at rest and immediately after a step-climbing exercise. The step was $23 \mathrm{~cm}$ high. The subjects completed the designed exercise workload and the signals of cardiac cycle and cardiac contractility were simultaneously collected and recorded by CCM. There are many exercise workload protocols for PCGET. A specific multistage incremental protocol was used, which had 4 stages. Exercise test was performed starting at an initial workload of $1750 \mathrm{~J}$. Each subsequent stage had an increment of $1750 \mathrm{~J}$. The peak workload was $7000 \mathrm{~J}$. There were 1-minute intervals between stages. The subjects repeatedly mounted and descended the step. The required number of times of step-climbing was calculated from the target workload, step height, and the subjects' body weight. The end point of the exercise test was usually limited by subject symptoms, or by achievement of the designed exercise workload. The time taken for completing the workloads 1750 J, $3500 \mathrm{~J}, 5250 \mathrm{~J}$, and $7000 \mathrm{~J}$ was recorded, respectively. During recovery after exercise, heart sound signals were recorded immediately after exercise, and at $1,5,10,15$, 20, 25, and 30 minutes after exercise. The ratio of the amplitude of the first heart sound to the amplitude of the second heart sound (S1/S2) on the same phonocardiogram was calculated from the measured data.

\subsection{Basic Method for Signal Measurement and Analysis}

Heart sound signal was converted several times during processing, and finally, it was converted to screen coordinates to construct a waveform graph. The basic points concerning heart sound quantitative analysis were: 1) measuring the duration and the amplitude of relevant heart sound components; 2) calculating and analyzing 
relevant indicators based on the data obtained from the above measurements, including S1/S2 ratio, $\mathrm{HR}$, and power (unit: W). Since the thickness of the chest wall of different subjects is various, the absolute amplitude of S1 cannot be used as an indicator for evaluating cardiac contractility. So, the design of some relative value indicators for evaluating cardiac reserve is very important, such as $\mathrm{S} 1 / \mathrm{S} 2$ ratio, D/S ratio, etc, which have dimensionless values. To measure and evaluate heart rate and cardiac contractility change trend, a self-control trial was designed.

\subsection{Construction of Cardiac Reserve Mobilization Trend Graph}

In order to show the cardiac reserve mobilization trend during exercise, $x-y$ plots were constructed from the measured data (employing the mean values). S1/S2 ratio, $\mathrm{HR}$, and power (vertical coordinates) were plotted against the work done during exercise (marked on the horizontal axis). The points of S1/S2 ratio, HR, and power corresponding $1750 \mathrm{~J}$ were connected to the points of their baseline value, producing 3 lines, S1/S2 line, HR line, and power line (W line), which, then connected to their own various points corresponding to 3500 J, $5250 \mathrm{~J}$, and $7000 \mathrm{~J}$, obtaining 3 polygonal lines, respectively. A higher slope value indicates a steeper incline. In order to show the cardiac function recovery course, a graph was constructed to the right of the graph of cardiac reserve mobilization trend, the vertical axes of which were the same as the left panel, and the horizontal axis changed to a time axis marked with the time points of cardiac function recovery.

\subsection{Statistical Analysis}

Quantitative data are presented as mean \pm SD. Statistical comparisons between 2 groups were performed with corresponding t test. A value of $P<0.05$ was considered to be statistically significant. Statistical analysis was performed with SPSS software (SPSS Inc).

\section{RESULTS}

The data of cardiac reserve mobilization trend of two groups are shown in Table 1.

The data of the recovery course of two groups are shown in Table 2.

The basic demographic data of the two groups are shown in Table 3.

Cardiac reserve mobilization trend and the recovery course of athletes are shown in Figure 1.

Cardiac reserve mobilization trend and the recovery course of non-athletes are shown in Figure 2.

\section{DISCUSSION}

Figure 1 shows the characteristics of the cardiac reserve
Table 1. The data of cardiac reserve mobilization trend of two groups.

\begin{tabular}{|c|c|c|c|}
\hline & Group A & Group B & $P$ value \\
\hline \multicolumn{4}{|l|}{$0 \mathrm{~J}$} \\
\hline $\begin{array}{c}\mathrm{S} 1 / \mathrm{S} 2 \\
\mathrm{HR}\end{array}$ & $\begin{array}{c}1.66 \pm 0.82 \\
87 \pm 10\end{array}$ & $\begin{array}{c}1.36 \pm 0.72 \\
64 \pm 13\end{array}$ & $\begin{array}{l}0.477 \\
0.003\end{array}$ \\
\hline \multicolumn{4}{|l|}{$1750 \mathrm{~J}$} \\
\hline $\begin{array}{c}\mathrm{S} 1 / \mathrm{S} 2 \\
\mathrm{HR} \\
\mathrm{W}\end{array}$ & $\begin{array}{c}3.66 \pm 1.57 \\
133 \pm 31 \\
125.43 \pm 41.98\end{array}$ & $\begin{array}{c}4.22 \pm 2.71 \\
121 \pm 22 \\
179 \pm 35.97\end{array}$ & $\begin{array}{l}0.642 \\
0.446 \\
0.025\end{array}$ \\
\hline \multicolumn{4}{|l|}{$3500 \mathrm{~J}$} \\
\hline $\begin{array}{c}\text { S1/S2 } \\
\text { HR } \\
\text { W }\end{array}$ & $\begin{array}{c}6.76 \pm 3.16 \\
162 \pm 22 \\
123 \pm 27.71\end{array}$ & $\begin{array}{c}8.76 \pm 3.65 \\
154 \pm 13 \\
183.57 \pm 50.75\end{array}$ & $\begin{array}{l}0.294 \\
0.458 \\
0.021\end{array}$ \\
\hline \multicolumn{4}{|l|}{$5250 \mathrm{~J}$} \\
\hline $\begin{array}{c}\text { S1/S2 } \\
\text { HR } \\
\text { W }\end{array}$ & $\begin{array}{c}6.7 \pm 2.16 \\
177 \pm 19 \\
118.29 \pm 31.89\end{array}$ & $\begin{array}{c}7.1 \pm 2.67 \\
166 \pm 9 \\
163.71 \pm 57.55\end{array}$ & $\begin{array}{l}0.763 \\
0.216 \\
0.093\end{array}$ \\
\hline \multicolumn{4}{|l|}{$7000 \mathrm{~J}$} \\
\hline $\begin{array}{c}\text { S1/S2 } \\
\text { HR } \\
\text { W }\end{array}$ & $\begin{array}{c}7.35 \pm 1.78 \\
180 \pm 11 \\
105.71 \pm 40.43\end{array}$ & $\begin{array}{c}8.54 \pm 1.04 \\
170 \pm 8 \\
163.57 \pm 42.38\end{array}$ & $\begin{array}{l}0.153 \\
0.066 \\
0.023\end{array}$ \\
\hline
\end{tabular}

Table 2. The data of the recovery course of two groups.

\begin{tabular}{cccc}
\hline & Group A & Group B & $P$ value \\
\hline 1 min & & & \\
\hline S1/S2 & $5.96 \pm 2.8$ & $5.87 \pm 2.95$ & 0.951 \\
HR & $152 \pm 10$ & $154 \pm 9$ & 0.739 \\
5 min & & & \\
S1/S2 & $2.56 \pm 1.48$ & $1.9 \pm 0.77$ & 0.316 \\
HR & $122 \pm 11$ & $121 \pm 18$ & 0.234 \\
10 min & & & \\
S1/S2 & $2.41 \pm 1.89$ & $1.61 \pm 0.48$ & 0.298 \\
HR & $127 \pm 30$ & $102 \pm 17$ & 0.076 \\
15 min & & & \\
S1/S2 & $2.39 \pm 0.93$ & $1.46 \pm 0.51$ & 0.043 \\
HR & $117 \pm 15$ & $100 \pm 14$ & 0.042 \\
20 min & & & \\
S1/S2 & $3.04 \pm 2.31$ & $1.37 \pm 0.6$ & 0.298 \\
HR & $109 \pm 12$ & $94 \pm 12$ & 0.036 \\
25 min & & & \\
S1/S2 & $2.05 \pm 1.55$ & $1.37 \pm 0.6$ & 0.298 \\
HR & $108 \pm 11$ & $94 \pm 12$ & 0.036 \\
30 min & & & \\
S1/S2 & $2.49 \pm 2.03$ & $1.16 \pm 0.41$ & 0.115 \\
HR & $111 \pm 17$ & $93 \pm 11$ & 0.039 \\
\hline
\end{tabular}

mobilization trend and recovery of athletes (Group B). HR and S1/S2 ratio increase with the increase in workload from $1750 \mathrm{~J}$ to $7000 \mathrm{~J}$, but power output gradually decreases, which is obviously related to the slowing of step-climbing, suggesting that skeletal muscle starts to fatigue. In the right part of the Figure 1, the power line 
Table 3. Basic demographic data of the two groups.

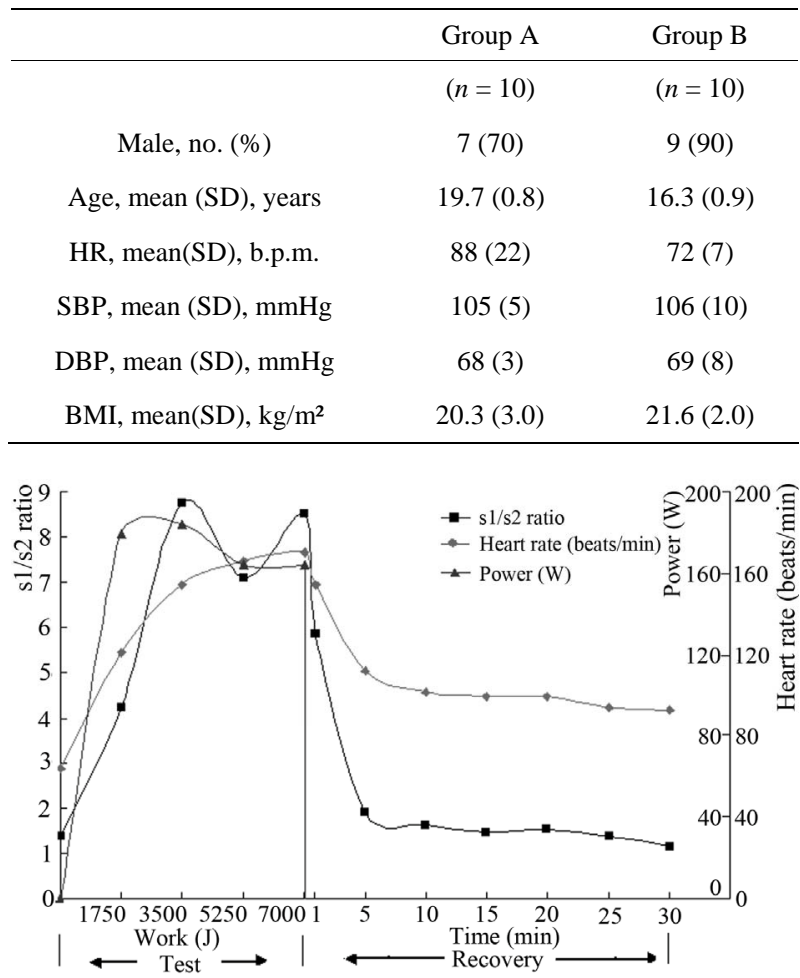

Figure 1. Cardiac reserve mobilization trend and the recovery course of athletes.

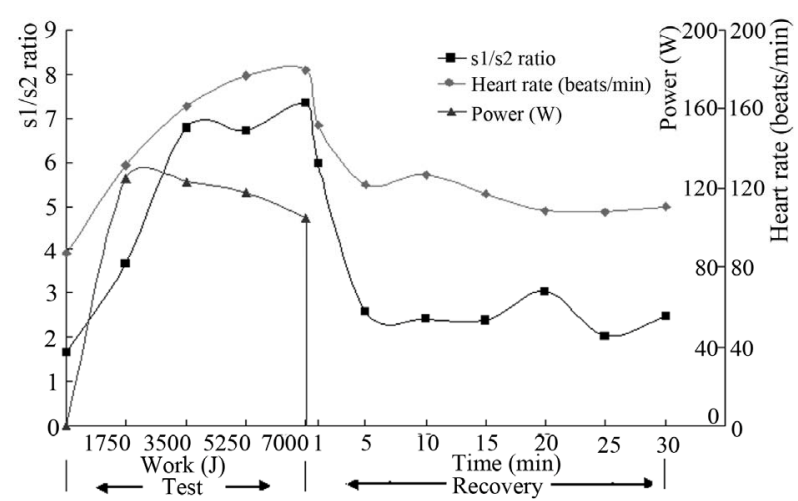

Figure 2. Cardiac reserve mobilization trend and the recovery course of non-athletes.

turns downward and reverts back to the horizontal axis when abrupt cessation of exercise, then, the S1/S2 line and HR line also go downward. The S1/S2 line reverts back to the baseline level 25 min after cessation of exercise, whereas the HR line has not yet reverted back to the baseline level $30 \mathrm{~min}$ after cessation of exercise, although HR has decreased below 100 beats/min.

Figure 2 shows the characteristics of the cardiac reserve mobilization trend and recovery of general students (Group A), which is similar to Figure 1. In the right part of the Figure 2, the power line turns downward and reverts back to the horizontal axis when abrupt cessation of exercise, the S1/S2 line and HR line also go downward, but until 30 min after cessation of exercise, both S1/S2 line and HR line do not revert back to the baseline level, S1/S2 remains above 2 and HR above 100 beats/ min.

By comparing Figure 1 with Figure 2, some different features of the cardiac reserve mobilization can be found between two groups. The level of S1/S2 ratio of the athletes is higher than that of the general students, which indicates that the athletes have more potential of cardiac reserve. The level and speed of increase of power output of the athletes are higher than the general students; power done by the general students decreases earlier than the athletes, which indicates that the skeletal muscle of the general students is more likely to be tired than the athletes. The HR recovery of the general students is slower than athletes. It would be due to the effect of long-term training so that the level and speed of increase of power output of the athletes are higher than the general students and the athletes maintain their S1/S2 ratio at a higher level longer than the general students.

The recovery course shown in Figures $\mathbf{1}$ and $\mathbf{2}$ indicates that the cardiac contractility recovery is earlier than the HR recovery.

With concrete data, Tables 1 and $\mathbf{2}$ show that 1) downsloping of power line of the general students after doing $1750 \mathrm{~J}$ of work, whereas the down-sloping of power line of the athletes after doing $3500 \mathrm{~J}$ of work; 2) for the athletes, the S1/S2 ratio recovery approaching to baseline value at $15 \mathrm{~min}$ after exercise cessation, whereas for the general students, at 25 min after exercise cessation; 3) at 30 min after exercise cessation, HR recovery of the athletes already to $<100$ beats/min, whereas for the general students, still above 100 beats/min.

These results indicate that cardiac reserve mobilization trend graph can reveal the extent and speed of cardiac function up-regulation during physical activities. Simultaneous measurement of both cardiac reserve mobilization trend and power output during exercise might be beneficial to comprehensive evaluation of cardiac function.

The reasons for incorporating $\mathrm{W}$ line in cardiac reserve mobilization trend graph are that 1) $\mathrm{W}$ line can show the corresponding power of the values of S1/S2 ratio and HR; 2) a higher power value implies a higher explosive power, i.e., doing much more work during a short period of time.

Most of the subjects cannot maintain a high level of power during the entire exercise test. Often the highest power occurs during the first stage of exercise test, when completing the workload of $1750 \mathrm{~J}$ (in our study protocol), since skeletal muscle fatigue is before cardiac fatigue. Shifting downward trend of $\mathrm{W}$ line in this graph represents skeletal muscle fatigue of limbs and trunk, 
mainly of legs.

A previous study by our team [17] suggested that high level of cardiac reserve of athletes was mainly cardiac contractility reserve rather than heart rate reserve. There was also a significant difference of power output between athletes and non-athletes, which suggested that athletes can mobilize their cardiac contractility reserve more rapidly than non-athletes.

In brief, this method for evaluating cardiac function up-regulation and recovery course is safe, easy, reliable, effective, which is beneficial for athlete selection, training, and matchmaking.

\section{REFERENCES}

[1] Kokkinos, P., Myers, J., Faselis, C., Panagiotakos, D.B., Doumas, M., Pittaras, A., et al. (2010) Exercise capacity and mortality in older men: A 20-year follow-up study. Circulation, 122, 790-797. doi:10.1161/CIRCULATIONAHA.110.938852

[2] Myers, J., Prakash, M., Froelicher, V., Do, D., Partington, S. and Atwood, J.E. (2002) Exercise capacity and mortality among men referred for exercise testing. The New England Journal of Medicine, 346, 793-801. doi:10.1056/NEJMoa011858

[3] Cooke, G.A., Marshall, P., Al-Timman, J.K., Wright, D.J., Riley, R., Hainsworth, R., et al. (1998) Physiological cardiac reserve: Development of a non-invasive method and first estimates in man. Heart, 79, 289-294.

[4] Watanabe, J., Thamilarasan, T., Blackstone, E.H., Thomas, J.D. and Lauer, M.S. (2001) Heart rate recovery immediately after treadmill exercise and left ventricular systolic dysfunction as predictors of mortality: The case of stress echocardiography. Circulation, 104, 1911-1916.

[5] Jolly, M.A., Danielle, M., Brennan, D.M. and Cho, L. (2011) Impact of exercise on heart rate recovery. Circulation, 124, 1520-1526. doi:10.1161/CIRCULATIONAHA.110.005009

[6] Durand, L.G., Langlois, Y.E., Lanthier, T., Chiarella, R., Coppens, P., Carioto, S., et al. (1990) Spectral analysis and acoustic transmission of mitral and aortic valve closure sounds in dogs. Part 4: Effects of modulating cardiac inotropy. Medical and Biological Engineering and Computing, 28, 439-445. doi:10.1007/BF02441967

[7] Hansen, P.B., Luisada, A.A., Miletich, D.J. and Albrecht, R.F. (1989) Phonocardiography as a monitor of cardiac performance during anesthesia. Anesthesia and Analgesia, 68, 385-387. doi:10.1213/00000539-198903000-00037
[8] Bombardini, T., Gemignani, V., Bianchini, E., Venneri, L., Petersen, C., Pasanisi, E., et al. (2008) Arterial pressure changes monitoring with a new precordial noninvasive sensor. Cardiovascular Ultrasound, 6, 41. doi:10.1186/1476-7120-6-41

[9] Hsieh, B.P., Unver, K., McNulty, E. and Schiller, NB. (2010) The amplitude ratio of the first to second heart sound is reduced in left ventricular systolic dysfunction. International Journal of Cardiology, 145, 133-135. doi:10.1016/j.ijcard.2009.06.060

[10] Chen, L., Tian, X., Xiao, S. and Peng, C. (2004) Comparative study of cardiac contractility recovery trend and heart rate recovery trend based on simultaneously telemetering cardiac contractility and heart rate signals after exercise. Acta Biophysica Sinica, 20, 403-407.

[11] Keir, P.J., Jamnik, V.K. and Gledhill, N. (2003) Technical-methodological report: A nomogram for peak leg power output in the vertical jump. Journal of Strength and Conditioning Research, 17, 701-703.

[12] Siebenmann, C., Robach, P., Jacobs, R.A., Rasmussen, P., Nordsborg, N.B., Díaz, V., et al. (2011) Live high-train low using normobaric hypoxia: A double-blinded, placebo-controlled study. Journal of Applied Physiology, 112, 106-117. http://www.ncbi.nlm.nih.gov/pubmed/22033534

[13] Adachi, H., Koikef, A., Obayashi, T., Umezawai, S., Aonumat, K., Lnada, M., et al. (1996) Does appropriate endurance exercise training improve cardiac function in patients with prior myocardial infarction? European Heart Journal, 17, 1511-1521. doi:10.1093/oxfordjournals.eurheartj.a014715

[14] Williams, S.G., Cooke, G.A., Wright, D.J., Parsons, W.J., Riley, R.L., Marshall, P., et al. (2001) Peak exercise cardiac power output: A direct indicator of cardiac function strongly predictive of prognosis in chronic heart failure. European Heart Journal, 22, 1496-1503. doi:10.1053/euhj.2000.2547

[15] Xiao, S., Wang, Z. and Hu, D. (2002) Studying cardiac contractility change trend to evaluate cardiac reserve. IEEE Engineering in Medicine and Biology Magazine, 21, 7476. doi:10.1109/51.993198

[16] Xiao, S., Guo, X., Sun, X. and Xiao, Z. (2002) A relative value method for measuring and evaluating cardiac reserve. Biomedical Engineering Online, 1, 6. http://www.ncbi.nlm.nih.gov/pmc/articles/PMC149375 doi:10.1186/1475-925X-1-6

[17] Wu, J., Lang, Y., Wang, F., Xiao, S. and Zhan, Z. (2003) A study of a method for simultaneous measurement of heart rate and cardiac contractility of athletes. Sports Science, 23, 107-120. 